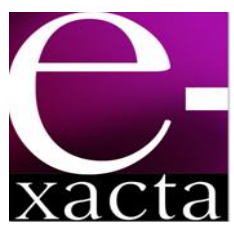

ISSN: 1984-3151

\section{ProduÇão de CARVÃo ATIVADO A PARTIR DE LODO DE ESGOTO DOMÉSTICO E SUA APLICAÇÃO NA ADSORÇÃO DO CORANTE PRETO REMAZOL 5 EM SOLUÇÃO AQUOSA}

\author{
PRODUCTION OF ACTIVATED CARBON FROM SEWAGE SLUDGE AND ITS \\ APPLICATION IN THE ADSORPTION OF THE REMAZOL BLACK DYE 5 \\ FROM AQUEOUS SOLUTION
}

\author{
1 Bacharel em Engenharia Ambiental. UESB, 2009. Mestre \\ em Engenharia Civil e Ambiental - PPGECEA/UEFS. \\ Doutorando em Engenharia de Minas, Metalúrgica e de \\ Materiais - PPGE3M/UFRGS, Porto Alegre. \\ glaydson.simoes@ufrgs.br. \\ 2 Doutor. UFSCAR, 1998. Professor da UFRGSI. Porto \\ Alegre, Rio Grande do Sul. profederlima@gmail.com. \\ 3 Doutor. Rheinisch Westfaelische Technische Hochschule \\ Aachen, 1987. Professor da UFRGS. Porto Alegre, Rio \\ Grande do Sul. sampaio@ufrgs.br.
}

Glaydson Simões dos Reis ${ }^{1}$; Eder Claudio Lima ${ }^{2}$; Carlos Hoffman Sampaio ${ }^{3}$

Recebido em: 21/03/2015 - Aprovado em: 23/11/2015 - Disponibilizado em: 30/11/2015

Resumo: O presente estudo investigou a viabilidade do lodo de esgoto como matéria- prima para produção de carvões ativados (CAs) para remoção do corante preto remazol 5 de soluções aquosas. Duas amostras foram preparadas; um carvão quimicamente ativado com $\mathrm{ZnCl}_{2}$ denominado $\mathrm{CAZ}$ e um carvão ativado sem ativação química denominado CA. Os CAs foram caracterizados por adsorção/dessorção de $N_{2}$, área superficial especifica $S_{B E T}$ e Microscopia eletrônica por varredura (MEV). De acordo com os resultados obtidos pelas isotermas de $N_{2}$ os carvões foram classificados como mesoporosos, no entanto a amostra CAZ apresentou elevado valor de BET comparado ao carvão CA, 612 e 21,8 $\mathrm{m}^{2} \mathrm{~g}^{-1}$, respectivamente. A habilidade dos CAs na remoção do corante $R B-5$ foi investigado empregando processo de adsorção em batelada. Os dados de equilíbrio foram ajustados aos modelos de isotermas de Lagmuir, freundlich e Sips. Levando em conta os valores de $R^{2}$ e função erro (SD), os dados foram melhores ajustados à isoterma de Sips. A capacidade máxima adsorvida do corante RB-5 sobre os carvões CA e CAZ foram de 16.12 e $156.64 \mathrm{mgg}^{-1}$, respectivamente.

PALAVRAS-CHAVE: Lodo de esgoto. Ativação química. Poluentes orgânicos. Isotermas de adsorção. Tratamento de efluente.

Abstract: The present study investigated the feasibility of sewage sludge as raw material for production of activated carbon to remove Remazol Black 5 dye from aqueous solutions. Two activated carbons were prepared; activated carbon chemically with $\mathrm{ZnCl}_{2}$ named $\mathrm{CAZ}$ and activated carbon without activation named CA. The adsorbents were characterised using $N_{2}$ adsorption/desorption isotherms, specific surface area $\left(S_{B E T}\right)$ and scanning electron microscopy. According to the $\mathrm{N}_{2}$ isotherms both samples were characterised as mesoporous activated carbons, however the sample $A C Z$ presented higher $S_{B E T}$ compared to the sample CA, 612 and $21,8 \mathrm{~m}^{2} \mathrm{~g}^{-1}$, respectively. The ability of activated carbons to remove the $R B-5$ was investigated using batch adsorption procedure. The equilibrium data were fitted to Langmuir, Freundlich and Sips isotherm models. Taking into account the determination coefficient $\left(R^{2}\right)$ and error function $(S D)$, the data were best fitted to Sips isotherm model. The maximum adsorption capacities of RB-5 onto $A C$ and $A C Z$ are 16.12 and $156.64 \mathrm{mgg}^{-1}$, respectively.

KEYWORDS: Sewage sludge. Chemical activation. Organic pollutant. Adsorption isotherms. Wastewater treatment. 


\section{INTRODUÇÃo}

As atividades industriais são responsáveis por gerar grande volume de espécies nocivas em efluentes de águas residuais, entre essas espécies estão os corantes. Como consequência, uma grande quantidade de rejeitos, sem tratamento, é despejada em águas naturais causando grandes problemas ambientais. Tais problemas estão relacionados ao fato dos corantes normalmente serem recalcitrantes, ou apresentarem cinética de degradação lenta para processos biológicos convencionais, resultando em efluentes finais com coloração ainda muito intensa (CARDOSO et al., 2011a; ROYER et al., 2010). A presença de corantes nos efluentes é altamente visível, afeta a estética, a transparência da água e a solubilidade de gases nos corpos receptores, reduzindo também a capacidade de regeneração dos corpos hídricos em função da redução da penetração de luz solar e consequente alteração dos processos de fotossíntese (CARNEIRO et al., 2010).

Face a isso, se faz necessário tratar os efluentes antes de serem dispostos nos mananciais. Dentre os tratamentos de efluentes contendo elevadas concentrações de corantes, destacam-se a oxidação biológica, os processos envolvendo membranas, os processos oxidativos avançados, a coagulação e a adsorção (RIBAS et al., 2014; KANNAN, MUTHURAJA, DEVI, 2013). Alguns desses métodos apresentam algumas desvantagens como a coagulação, que se baseia na adição de substâncias químicas e que geram problemas operacionais devido ao alto custo. O método de adsorção em carvão ativado é um processo que tem sido examinado como alternativa tecnológica devido à sua simplicidade, eficiência e disponibilidade de adsorventes de baixo custo (ARZU et al., 2013; NOREEN et al., 2013).

Uma variedade de adsorventes tem sido empregada no tratamento de efluentes contento corante, pela técnica de adsorção, destacando-se sílica
(HONGSAWATA et al., 2014), alumina (KANNAN, MUTHURAJA, DEVI, 2013), celulose (ADEBAYO et al., 2014) e carvão ativo (KEARNS et al., 2014).

O carvão ativado é um material que apresenta boas características como alta capacidade de adsorção, baixa reatividade térmica, resistência a grandes variações de $\mathrm{pH}$ no meio e capacidade de regeneração, porém ainda é considerado um produto caro. Então, pesquisas recentes têm investigado a capacidade adsortiva de materiais de baixo-custo como lodo residual do biogás, vermiculita, quitosana, zeolitas, lamas de fundo, argilas vermelhas e lodo de esgoto (VIJAYARAGHAVAN et al., 2008; KARADAG et at., 2007). Seguindo esse propósito, este trabalho tem como objetivo avaliar a eficiência de remoção do corante preto remazol 5 utilizando como adsorvente alternativo carvões ativados produzidos a partir de lodo de esgoto doméstico.

\section{MATERIAIS E MÉtodos}

\subsection{SOLUÇÕES E REAGENTES}

Utilizou-se o corante preto remazol (RB-5, com 55\% de pureza), adquirido da Sigma-Aldrich. Uma solução estoque $\left(1,00 \mathrm{gL}^{-1}\right)$ do corante foi previamente preparada por pesagem e posteriormente diluída para se obter as soluções de trabalho. $\mathrm{O}$ pH das soluções foi fixada em pH entre 6 e 7 .

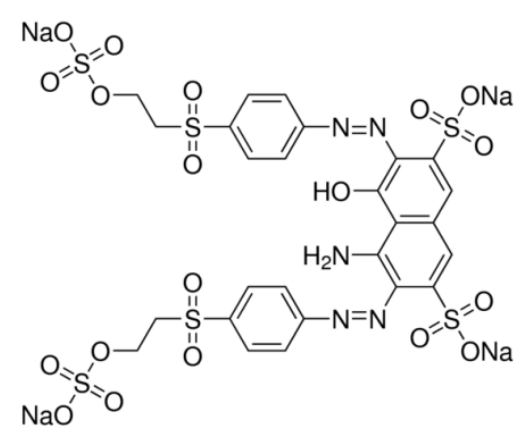

Figura 1 - Fórmula estrutural do corante preto remazol 5 Fonte: Próprio autor. 


\subsection{ADSORVENTE}

O lodo de esgoto sanitário foi gerado e coletado na ETE Navegantes, localizada no bairro São João Navegantes, no município de Porto Alegre. Para o preparo do carvão de lodo de esgoto foram utilizados $10,0 \mathrm{~g}$ de lodo de esgoto em pó, $10,0 \mathrm{~g}$ de cloreto de zinco e 10,0 mL de água que foram misturados para se obter uma pasta homogênea. A pasta resultante foi colocada em cadinho de porcelana e seco a temperatura ambiente durante $24 \mathrm{~h}$. Após seco, o cilindro foi colocado em um reator de aço inoxidável e aquecido a uma taxa de $10{ }^{\circ} \mathrm{Cmin}^{-1}$ até $800{ }^{\circ} \mathrm{C}$, durante $60 \mathrm{~min}$. sob fluxo de nitrogênio $\left(25 \mathrm{mLmin}^{-1}\right)$. $\mathrm{O}$ adsorvente foi resfriado até temperatura ambiente também sob fluxo de nitrogênio. Posteriormente, 0 material resultante foi moído e peneirado com tamanho de partículas $\leq 90 \mu \mathrm{m}$. Com a finalidade de aumentar a capacidade de adsorção, uma ativação química foi realizada no adsorvente. A ativação constituiu em colocar $10 \mathrm{~g}$ do carvão produzido em contato com $160 \mathrm{~mL}$ de $\mathrm{HCl} 1 \mathrm{molL}^{-1}$, a mistura foi agitada com um agitador magnético e permaneceu em refluxo durante $4 \mathrm{~h}$ a uma temperatura de $70{ }^{\circ} \mathrm{C}$. $\mathrm{O}$ material foi filtrado e lavado exaustivamente com água destilada para remover os compostos inorgânicos do adsorvente, posteriormente foi seco a $105^{\circ} \mathrm{C}$ durante $5 \mathrm{~h}$ e armazenado adequadamente até sua utilização. $O$ adsorvente foi nomeado CAZ (carvão ativado com zinco e lavado com acido clorídrico $1 \mathrm{molL}^{-1}$ ) e CA (carvão ativado sem utilização de zinco e sem lavagem acida).

O rendimento do processo de síntese dos carvões ativados, a partir do lodo, foi calculado através da Eq. 1.

$\mathrm{R}(\%)=\left(\mathrm{m}_{\mathrm{c}} / \mathrm{m}_{\mathrm{p}}\right) \cdot 100$

Em que: $\mathrm{R}$ é o rendimento de carvão (\%), $\mathrm{m}_{\mathrm{c}}$ é a massa do carvão obtido $(g)$ e $m_{p}$ é a massa do lodo precursor (g).

\subsection{CARACTERIZAÇÃo dos ADSORVENTES}

Para a determinação da área superficial e distribuição do volume dos poros dos CAs, isotermas de adsorção e dessorção de $\mathrm{N} 2$ foram realizadas a $77 \mathrm{~K}$, em um Autosorb-1 (Quantachrome). A distribuição do tamanho dos poros foi calculada pelo método $\mathrm{BJH}$, a partir da isoterma de adsorção. A área superficial foi calculada utilizando a equação BET.

A morfologia dos carvões ativos foi avaliada por microscopia eletrônica de varredura (MEV), em um equipamento Shimadzu (ZeissMA10). A amostra foi colocada em um suporte de alumínio e uma fina camada de ouro foi aplicada em um evaporador Quorum Technologies-Q150R.

\subsection{AdSORÇÃo EM BATELADA}

Ensaios de adsorção foram realizados utilizando quantidades de adsorventes que variaram de 10,0 a $100,0 \mathrm{mg}$ em contato com $20 \mathrm{~mL}$ da solução de corante RB- 5 nas concentrações iniciais que variaram entre 5,0 e $1000,0 \mathrm{mgL}^{-1}$. As amostras foram colocadas em tubos Falcon de $50 \mathrm{~mL}$ e agitadas em um shaker magnético, o tempo de agitação variou entre 5 e $480 \mathrm{~min}$. Para os testes de $\mathrm{pH}$ foi fixado em 6,0. Após os experimentos as amostras foram centrifugadas por $10 \mathrm{~min}$ a $5.000 \mathrm{rpm}$ para separar os adsorventes das soluções de corantes. Para a quantificação do RB-5 residual das amostras a solução foi analisada em espectrofotômetro UV/VIS (597 nm). Quando necessário, as alíquotas foram diluídas com água deionizada antes da medição espectroscópica. A quantidade de corante adsorvido foi calculada usando a Eq. 2.

$$
q=\frac{\left(C_{o}-C_{f}\right)}{m} . V
$$




\subsection{AJUSTE dOS MODELOS MATEMÁTICOS}

Os dados cinéticos e de equilíbrio foram ajustados através de métodos não lineares com interações sucessivas calculadas pelo método de LevenbergMarquardt. Essas interações também foram avaliadas com o auxílio do método Simplex, com base no ajuste não linear do software Microcal Origin 9.0. Um coeficiente de determinação $\left(R^{2}\right)$, um coeficiente de determinação ajustado ( $R^{2}$ adj) e uma função de erro (SD) foram utilizados em conjunto para avaliar a adequação dos modelos (RIBAS et al., 2014; CARDOSO et al., 2011a). Um SD é definido como uma medida das diferenças entre os valores teóricos e experimentais de corante adsorvido. $O \mathrm{R}^{2}, \mathrm{R}^{2}$ adj e SD são descritos nas Eq. 3, 4 e 5, respectivamente.

$$
\begin{aligned}
& R^{2}=\left(\frac{\sum_{i}^{n}\left(q_{i, e x p}-\bar{q}_{i, \text { exp }}\right)^{2}-\sum_{i}^{n}\left(q_{i, \text { exp }}-q_{i, \text { model }}\right)^{2}}{\sum_{i}^{n}\left(q_{i, \text { exp }}-\bar{q}_{i, \text { exp }}\right)^{2}}\right) \\
& R_{a d j}^{2}=1-\left(1-R^{2}\right) \cdot\left(\frac{n-1}{n-p-1}\right) \\
& S D=\sqrt{\left(\frac{1}{n-p}\right) \cdot \sum_{i}^{n}\left(q_{i, \text { exp }}-q_{i, \text { model }}\right)^{2}}
\end{aligned}
$$

Nessas equações, $q_{i}$,model representa 0 valor de $q$ teórico individual previsto pelo modelo, $\mathrm{q}_{\mathrm{i} \text {,exp }}$ representa o valor de q experimental individual, $q_{\exp }$ é a média de $q$ experimental, $\mathrm{n}$ representa o número de experimentos, enquanto $p$ representa o número de parâmetros do modelo (RIBAS et al., 2014; CARDOSO et al., 2011a).

\subsection{MODELOS DE EQUILÍBRIO}

Os modelos utilizados no presente estudo foram Langmuir, Freundlich e Sips. A isoterma de Langmuir é frequentemente empregada para descrever o comportamento do equilíbrio da adsorção em sistemas monocomponentes. É um modelo teórico que considera adsorção em monocamada e um número finito de sítios ativos (Langmuir, 1918). A equação geral do modelo é dada pela Eq. 6 :

$q_{e}=\left(Q_{\max } \cdot K_{L} \cdot C_{e}\right) /\left(1+K_{L} \cdot C_{e}\right)$

em que $q_{e}$ é a quantidade adsorvida no equilíbrio $\left(m g g^{-1}\right), Q_{\max }$ é a capacidade máxima de adsorção do adsorvente $\left(\mathrm{mgg}^{-1}\right), \mathrm{K}_{\mathrm{L}}$ é a constante de equilíbrio de Langmuir $\left(\mathrm{Lmg}^{-1}\right)$ e Ce representa a concentração de equilíbrio de adsorbato $\left(\mathrm{mgL}^{-1}\right)$.

A isoterma de Freundlich é um modelo empírico que considera um adsorvente com superfície heterogênea e formação de multicamadas pelo soluto. O modelo de Freundlich é representado pela Eq. 7 (Freundlich, 1906):

$q_{e}=K_{f \cdot} C e^{1 / n_{F}}$

em que $\mathrm{K}_{\mathrm{f}}$ é a constante de equilíbrio do modelo de Freundlich (mg g-1) e nF é um expoente adimensional da equação de Freundlich.

O modelo de Sips combina os modelos de Langmuir e Freundlich, dada pela Eq. 8.

$q_{e}=\left(Q_{\max } \cdot K_{s} \cdot C_{e}{ }^{1 / n_{s}}\right) /\left(1+K_{s} \cdot C_{e}{ }^{1 / n_{s}}\right)$

Na qual $K_{s}$ é a constante de equilíbrio de adsorção de Sips $\left(\mathrm{mgL}^{-1}\right)^{-1 / n s}$ e $\mathrm{Q}_{\max }$ é a capacidade máxima de adsorção $\left(\mathrm{mgg}^{-1}\right)$, e $\mathrm{n}_{\mathrm{s}}$ é o expoente de Sips (adimensional). Em baixas concentrações de adsorvato esse modelo assume a forma de Freundlich, enquanto que em altas concentrações assume a forma de Langmuir em monocamadas.

\section{RESUltados E discussão}

\subsection{MiCROSCOPIA ELETRÔNICA DE VARREDURA}

A microscopia eletrônica de varredura foi uma técnica empregada para observar a morfologia dos materiais analisados. As micrografias apresentadas na FIG. 2 são referente às amostras $C A(A)$ e $C A Z(B)$. 

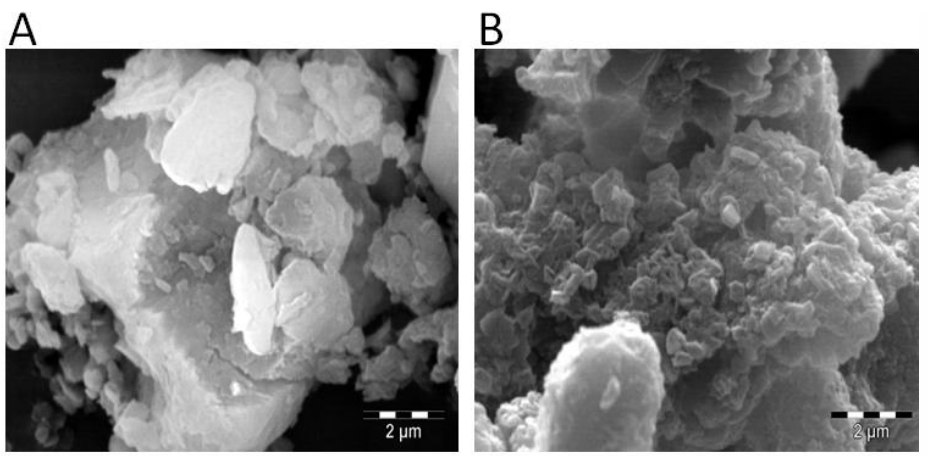

Figura 2 - Micrografia obtida por MEV do CA e CAZ Fonte: Próprio autor.

Observa-se pelas micrografias que após o processo de ativação (FIG. 2B) que houve uma modificação na estrutura do carvão ativo, com o aparecimento de uma superfície irregular e porosa mostrando que 0 processo de impregnação/lavagem ácida altera a morfologia do material com aparecimento de uma estrutura porosa.

\subsection{Propriedades texturais}

A FIG. 3 apresenta as isotermas de adsorção/dessorção de nitrogênio e a distribuição de poros dos carvões ativados obtidos do lodo de esgoto doméstico para as amostras CA e CAZ. Os perfis das curvas se assemelham ao Tipo IV da classificação das isotermas propostas por Brunauer típicos para materiais mesoporosos (BALBUENAT; GUBBINS, 1993). As isotermas apresentam condensação capilar em mesoporos e histerese.

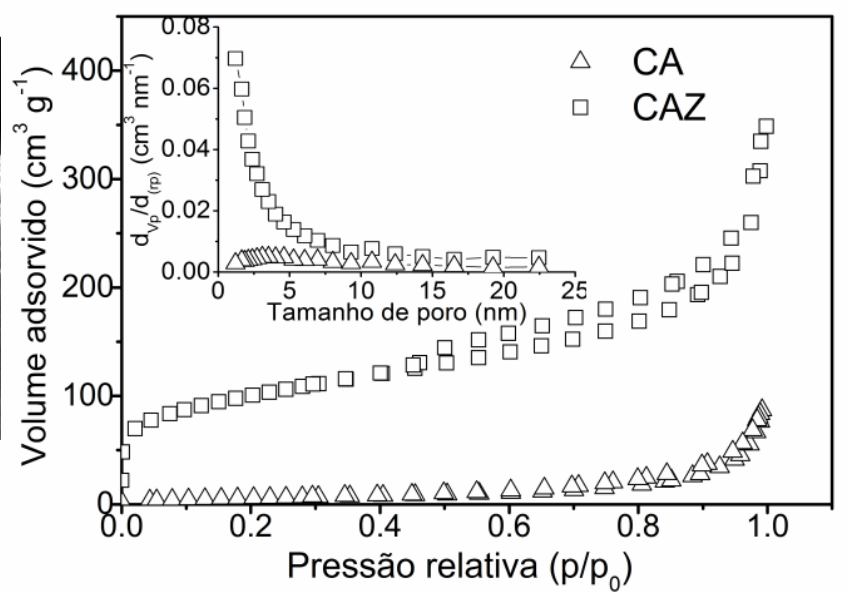

Figura 3 - Isotermas de adsorção/dessorção de $\mathrm{N}_{2} \mathrm{e}$ distribuição de poros dos carvões preparados

A partir das isotermas apresentadas na FIG. 3, foram determinados os valores de área $B E T$ ( $\left.S_{B E T}\right)$, volume de poro (V) e tamanho de poro (TAB. 1) sendo os valores $S_{\mathrm{BET}}$ encontrados de 1,82, 21,8 e $612 \mathrm{~m}^{2} \mathrm{~g}^{-1}$ para o lodo, carvão ativado sem e com ativação (CA e CAZ), respectivamente. Pode-se observar que a impregnação com cloreto de zinco e sua posterior lavagem acida mostraram-se eficiente na obtenção de carvão ativado com elevada área superficial específica, aumentando a $\mathrm{S}_{\mathrm{BET}}$ em $2800 \%$ $\left(612 \mathrm{~m}^{2} \mathrm{~g}^{-1}\right)$ na temperatura de $800 \stackrel{\circ}{\circ}$ contra $21,8 \mathrm{~m}^{2} \mathrm{~g}^{-1}$ para o carvão ativado $\mathrm{CA}$, sem ativação.

Tabela 1

Propriedades texturais do precursor e dos carvões ativados CA e CAZ obtidas pelo método BET

\begin{tabular}{c|c|c|c}
\hline Amostras & Lodo & CA & CAZ \\
\hline Parâmetro \\
\multicolumn{4}{|l}{} \\
\hline $\mathrm{S}_{\mathrm{BET}}\left(\mathrm{m}^{2} \mathrm{~g}^{-1}\right)$ & 1,82 & 21,8 & 612 \\
$\mathrm{~V}\left(\mathrm{~cm}^{3} \mathrm{~g}^{-1}\right)$ & 0,002 & 0,007 & 0,621 \\
Tamanho & & & \\
médio de poro & & & \\
$(\mathrm{mn})$ & 13,44 & 8,25 & 3,11 \\
Rendimento & - & 23,78 & 24,15 \\
\hline
\end{tabular}

Como pode-se observar, a distribuição de da curva dos diâmetros de poros é descendente, onde o máximo é apresentado em 1,52 nm e sua pequena quantidade de poros variando entre 1,52 e $22 \mathrm{~nm}$. Entretanto este material pode ser considerado micro e 
mesoporoso corroborado pelas curvas da isoterma de $\mathrm{N}_{2}$ (BALBUENAT; GUBBINS, 1993).

Os rendimentos encontrados para os carvões ativados CA e CAZ foram de 23,78 e $24,15 \%$, respectivamente (TAB. 1). Esses valores assemelham-se aos resultados encontrados por CHEN et al. (2015), que variaram entre 17,71 e $28,35 \%$, as diferenças nos valores dos rendimentos podem ser atribuídas as diferentes composições físico-químicas dos lodos precursores e também das diferentes condições de pirólises (CHEN et al.,2015).

\subsection{INFLUÊNCIA DA MASSA NA ADSORÇÃO DO CORANTE RB-5}

A maior quantidade adsorvida do RB- 5 foi obtido para a quantidade de massa de 50,0 mg (FIG. 4). Para quantidades superiores que este valor, a porcentagem da remoção do RB-5 permanece quase constante.

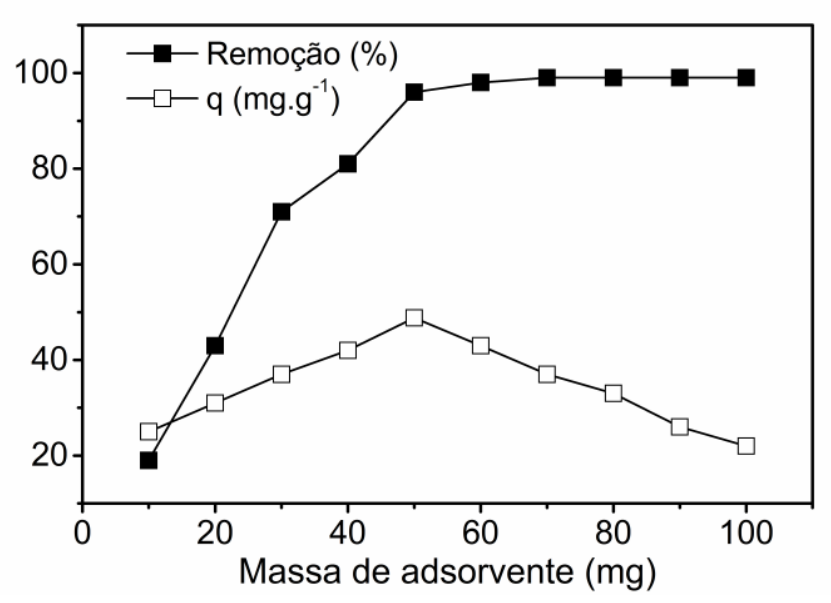

Figura 4 - Influência da massa na adsorção do corante RB-5

O aumento na porcentagem da remoção do RB- 5 com até $50,0 \mathrm{mg}$ de CA pode ser atribuído ao aumento da quantidade de sítios ativos disponíveis para adsorção, já reportando em vários trabalhos (SILVEIRA et al., 2014; CARDOSO et al., 2011a; ROYER et al.,2010). Por outro lado, o aumento na adição de massa de adsorvente promove uma notória diminuição na quantidade adsorvida por grama (q), (FIG. 4). A explicação pode ser atribuída a dois fatores. Primeiro, - aumento na massa de adsorvente quando a concentração e volume de RB-5 são fixados leva a saturação dos sítios ativos disponíveis para a adsorção. Segundo, a redução na capacidade adsortiva pode ser devido à agregação de partículas, resultante da alta quantidade de massa de adsorvente na solução. Face a isso, a quantidade de massa a ser utilizada nos testes de adsorção deve ser fixada em $50,0 \mathrm{mg}$, no qual é a massa que corresponde a mínima quantidade de adsorvente que leva a constante remoção do RB-5. A massa de adsorvente foi, então, fixada em $50,0 \mathrm{mg}$ em $20 \mathrm{~mL}$ de solução de RB-5.

\subsection{ESTUDO DE EQUILÍBRIO}

As isotermas de Langmuir, Freundlich e Sips foram utilizadas para avaliar e interpretar os dados experimentais. A FIG. 5 mostra a isoterma de adsorção na temperatura de $25^{\circ} \mathrm{C}$ para o corante RB5. Pode-se observar, através da FIG. 5 e TAB. 2, que o modelo de Sips descreve adequadamente os dados experimentais. 


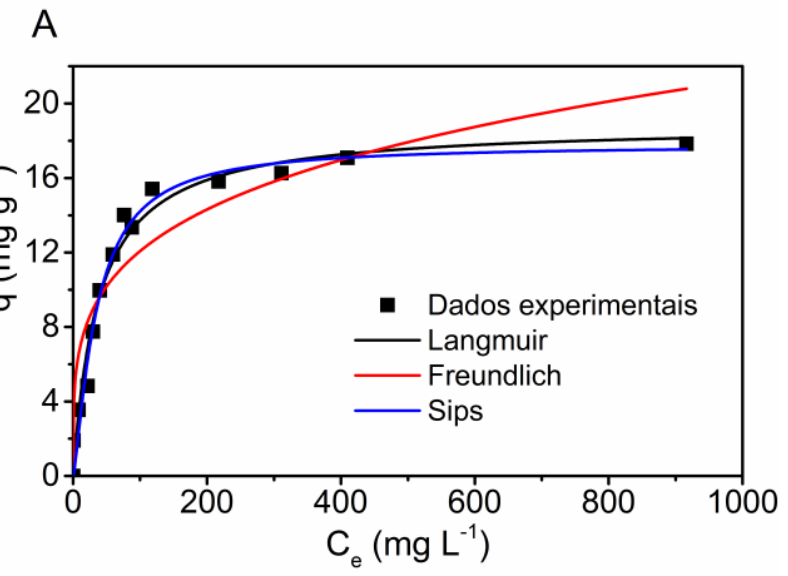

B

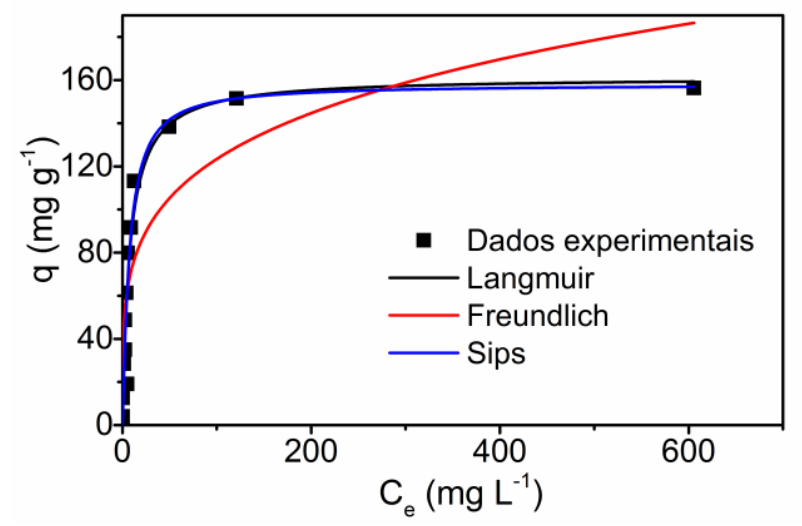

Figura 5 - Isotermas de adsorção do RB- 5 para as amostras CA (A) e CAZ (B) $(50 \mathrm{mg}$ de material; $20 \mathrm{~mL}$

de RB-5, tempo de contato $120 \mathrm{~min}, \mathrm{~T}=25^{\circ} \mathrm{C}$ )

A TAB. 02 apresenta os valores dos parâmetros e da função erro (SD) para todos os modelos analisados.

\section{Tabela 2}

Parâmetros das isotermas de adsorção para o corante RB-5 usando adorventes a base de lodo de esgoto $A C$ e $A C-Z$

\begin{tabular}{|c|c|c|}
\hline & CA & CAZ \\
\hline \multicolumn{3}{|l|}{ Langmuir } \\
\hline $\mathrm{Q}_{\max }\left(\mathrm{mgg}^{-1}\right)$ & 16,98 & 157,86 \\
\hline $\mathrm{K}_{\mathrm{L}}\left(\mathrm{Lmg}^{-1}\right)$ & $\begin{array}{c}0,0525 \\
0,985\end{array}$ & $\begin{array}{l}0,0289 \\
0,9948\end{array}$ \\
\hline$R^{2} a d j$ & 0,977 & 0,9871 \\
\hline $\mathrm{SD}\left(\mathrm{mgg}^{-1}\right)$ & 1,12 & 2,75 \\
\hline \multicolumn{3}{|l|}{ Freundlich } \\
\hline $\mathrm{K}_{\mathrm{F}}\left(\mathrm{mgg}^{-1}\left(\mathrm{mgL}^{-1}\right)^{-1 / 1 / F} \mathrm{~F}\right)$ & 1,186 & 15,421 \\
\hline$n_{F}$ & 1,775 & 4,856 \\
\hline $\mathrm{R}^{2}$ & 0,9054 & 0,8864 \\
\hline$R^{2}$ adj & 0,892 & 0,874 \\
\hline $\mathrm{SD}\left(\mathrm{mgg}^{-1}\right)$ & 5,84 & 6,54 \\
\hline
\end{tabular}

\begin{tabular}{c|c|c} 
Sips & \multicolumn{1}{l}{} \\
\hline $\mathrm{Q}_{\max }\left(\mathrm{mgg}^{-1}\right)$ & 16,12 & 156,64 \\
$\mathrm{~K}_{\mathrm{g}}\left(\mathrm{Lmg}^{-1}\right)$ & 1,102 & 1,984 \\
$\mathrm{n}_{\mathrm{s}}$ & 0,386 & 1,031 \\
$\mathrm{R}^{2}$ & 0,9941 & 0,9981 \\
$\mathrm{R}^{2} \mathrm{adj}$ & 0,972 & 0,9871 \\
$\mathrm{SD}\left(\mathrm{mgg}^{-1}\right)$ & 0,82 & 1,62 \\
\hline
\end{tabular}

Os valores da função erro SD do modelo de Freundlich variou entre 5,84-6,54, enquanto que para o modelo de Langmuir os valores variaram entre 1,122,75 . Baseados nos valores de $R^{2}, R^{2}$ adj e função erro SD, o modelo que melhor se ajustou aos dados experimentais foi o modelo de Sips (TAB. 2). O modelo de Sips apresentou os maiores valores para o $R^{2}$ e $R^{2}$ adj não obstante apresentou também os menores valores para a função erro $S D$, de acordo com a TAB. 2, o que significa que os valores de "q" obtidos pela isoterma de Sips são os mais próximos dos valores medidos experimentalmente. Em relação a isoterma de Langmuir os valores de $R^{2}, R^{2}$ adj e SD próximos ao do modelo de Sips indicando que o processo de adsorção ocorreu de maneira mais homogênea (LANGMUIR, 1918). Por outro lado, o modelo d Freundlich não obteve bom ajuste aos dados experimentais. A capacidade máxima de remoção (Qmax) para o modelo de Sips foi de 156,64 $\mathrm{mgg}^{-1}$ para a amostra submetida ao pré-tratamento com $\mathrm{ZnCl}_{2}$ e lavagem com $\mathrm{HCl}$ (CAZ).

\subsection{COMPARAÇÃO DA CAPACIDADE ADSORTIVA PARA O CORANTE RB-5 ENTRE DIFERENTES ADSORVENTES}

A TAB. 3 mostra a comparação na capacidade adsortiva do corante RB-5 entre diferentes adsorventes. Pode ser visto pela TAB. 3 que a amostra CAZ empregada neste trabalho mostrou elevada adsorção do corante RB-5 em relação a quatro dos sete diferentes adsorventes. Esse dado faz 
do lodo de esgoto, através da amostra CAZ, um excelente precursor para produzir adsorventes com elevada capacidade de adsorção, em especial na remoção do corante RB-5 de solução aquosa.

\section{Tabela 3}

Comparação da capacidade adsortiva do RB- 5 entre diferentes adsorventes

\begin{tabular}{c|c|c}
\hline \multicolumn{3}{c}{ Capacidade de adsorção/ mgg } \\
\hline Adsorvente & RB-5 & Referência \\
\hline Cinza leve & 7,93 & (EREN; ACAR, 2006) \\
(IP; BARFORD; MCKAY, \\
Carvão de osso & 157 & 2009) \\
& & (CARDOSO et al., \\
Talo de açai & 52,3 & 2011a) \\
Zeolita & 12,9 & $\begin{array}{c}\text { (KARADAG et al., 2007) } \\
\text { (CARDOSO et al., }\end{array}$ \\
$\begin{array}{c}\text { Casca de pinhão } \\
\text { (forma natural) }\end{array}$ & 74,6 & 2011b) \\
Alga marrom & 55,5 & (VIJAYARAGHAVAN; \\
YUN, 2008) \\
Bagaço de açúcar & 30 & (ARZU et al., 2013) \\
AC & 16,12 & This study \\
ACZ & 156,64 & This study \\
\hline
\end{tabular}

\section{CoNCLUSÃo}

O lodo de esgoto mostrou-se um bom precursor para produção de carvão ativado. Os carvões ativados CA e CAZ foram classificados como mesoporosos. O carvão submetido à ativação química (CAZ) apresentou área superficial elevada $\left(612 \mathrm{~m}^{2} \mathrm{~g}^{-1}\right) \mathrm{e}$ comparável com os descritos na literatura, assim como seu rendimento (24,15\%). A ativação química gerou um carvão com melhores propriedades adsortivas e sua capacidade máxima de adsorção (Qmax) para o corante RB-5, foi de 156,64 $\mathrm{mg} \mathrm{g}^{-1}$ frente a $17,12 \mathrm{mgg}^{-1}$ do carvão CA. Modelo de isoterma de Sips apresentou o melhor ajuste aos dados experimentais. Tais resultados indicam que 0 carvão produzido a partir do lodo de esgoto doméstico pode ser aplicado no tratamento de águas residuais reais, pois apresenta baixo custo e elevada capacidade de remoção.

\section{AgRADECIMENTOS}

Os autores agradecem ao CNPq pelo apoio financeiro.

\section{REFERÊNCIAS}

ADEBAYO, M, A. et al.. Adsorption of Procion Blue MX-R dye from aqueous solutionsby lignin chemically modified with aluminium and manganese. Journal of Hazardous Materials, Vol. 268, 43- 50, 2014.

ARZU, Y.; et al.. Kinetics of Remazol Black B adsorption onto carbon prepared from sugar beet pulp. Environmental Science Pollutant Resource, Vol.20, 2472-2483, 2013.

BALBUENAT, P.B. e GUBBINS, K.E. Theoretical Interpretation of Adsorption Behavior of Simple Fluids in Slit Pores. Langmuir, Vol.9, 1801-1814, 1993.

CARDOSO, N.F. et al.. Application of Aqai Stalks As Biosorbents for the Removal of the Dyes Reactive Black 5 and Reactive Orange 16 from Aqueous Solution. Journal Chemical Enginnering Data, Vol.56, 1857-1868, 2011a.

CARDOSO, N. F. et al.. Removal of remazol black B textile dye from aqueous solution by adsorption. Desalination, Vol.269, 92-103, 2011b.
CARNEIRO, P.A. et al.. Assessment of water contamination caused by a mutagenic textile effluent/dyehouse effluent bearing disperse dyes. Journal of Hazardous Materials, Vol. 174, 694-699, 2010.

CHEN, J. et al.. Removal of Dye Wastewater COD by Sludge Based Activated Carbon. Journal of Coastal Research, Vol. 73, 1-3, 2015.

EREN, Z. e ACAR, F.N. Adsorption of Reactive Black 5 from an aqueous solution: equilibrium and kinetic studies. Desalination,Vol.194, No.1-3, pp1-10, 2006.

FREUNDLICH, H. M. F. Über die adsorption in lösungen. In. Zeitschrift für Physikalische Chemie. Vol. 57, 385-470, 1906.

HONGSAWATA, P. et al.. Adsorption of ciprofloxacin on surface functionalized superparamagnetic porous silicas. Desalination and Water Treatment, Vol. 52, 4430-4442, 2014. 
IP, A.W.; BARFORD, J.P. e MCKAY, G. Reactive Black dye adsorption/desorption onto different adsorbents: effect of salt, surface chemistry, pore size and surface area. Journal Colloids Interface Science, Vol.337, 32-38, 2009.

KANNAN, C.; MUTHURAJA, K. e DEVI, M.R. Hazardous dyes removal from aqueoussolution over mesoporous aluminophosphate with textural porosity by adsorp-tion. Journal of Hazardous Materials, Vol. 244, 10-20, 2013.

KARADAG, D. et al.. Adsorption equilibrium and kinetics of Reactive Black 5 and Reactive Red 239 in aqueous solution onto surfactant-modified zeolite.

Journal of Chemical and Engineering data, Vol. 52, 1615-1620, 2007.

KEARNS, J,P. et al.. Adsorption to biochars: Effect of preparation conditions on equilibrium adsorption capacity and comparison with commercial activated carbon literature data. Water Research, Vol. 62, 2033, 2014.

LANGMUIR, I. The adsorption of gases on plane surfaces of glass, mica and platinum. Journal of the American Chemical Society. Vol. 40, 1361-1403, 1918.
NOREEN, $S$ et al.. Batch and fixed bed adsorption study for the removal of Drimarine Black CL-B dye from aqueous solution using a lignocellulosic waste: $\mathrm{A}$ cost affective adsorbent. Industries Crops and Products, Vol. 50 p. 568-579, 2013.

RIBAS, M.C. et al., Comparison of a homemade cocoa shell activated carbon with commercial activated carbon for the removal of reactive violet 5 dye from aqueous solutions. Chemical Engineering Journal, Vol. 248, 315-326, 2014.

ROYER, B. et al.. A useful organofunctionalized layered silicate for textile dye removal. Journal of Hazardous Materials, Vol. 181, 366-374, 2010.

SILVEIRA, M. B. et al.. Punica granatum Shell Preparation, Characterization, and Use for Crystal Violet Removal from Aqueous Solution. Clean - Soil Air Water, Vol. 42, 939-946, 2014.

VIJAYARAGHAVAN, K. e YUN, Y. S. Biosorption of C.I. Reactive Black 5 from aqueous solution using acid-treated biomass of brown seaweed Laminaria sp. Dyes and Pigments, Vol. 76, 726-732, 2008. 